\title{
Wireless Transmission of Friedlander-type Signals for the Dynamic Measurement of Blast Pressure
}

\author{
Mathieu Chalnot ${ }^{*}{ }^{[a]}$ Anthony Coustou, ${ }^{[a]}$ Hervé Aubert, ${ }^{[a]}$ Patrick Pons, ${ }^{[a]}$ Maylis Lavayssière, ${ }^{[b]}$ \\ Alexandre Lefrançois, ${ }^{[b]}$ Jérôme Luc, ${ }^{[b]}$
}

\begin{abstract}
During explosive detonation or air blast experiments, long cables are commonly used for transmitting signals delivered by pressure sensors to the safe place of the acquisition unit. While constant efforts are undertaken for enlarging the bandwidth of sensors in order to improve the measurement precision of the peak pressure, the limitations of the wired transmission of blast pressure signals delivered by these sensors are not addressed. We discuss here such limitations and propose a wireless new solution for the dynamic measurement of Friedlandertype signals delivered by ultra-wideband sensors (>10 MHz). Contrary to the wired solution, the distance between the sensor and the acquisition unit does not limit the measurement bandwidth of the wireless setup. Experimental results are reported for validation purposes and pave the way of the dynamic measurement of blast pressure variations occurring at the heart of the fireball generated during explosions.
\end{abstract}

Keywords: Wireless transmission, explosive, detonation, air blast, radiofrequency, dynamic pressure measurement

\section{Introduction}

During a high explosive detonation, a rapid amount of energy is released, generating a supersonic shock wave, with discontinuities in pressure, gas density, temperature and particle velocity across the wave front. At the shock wave front, the pressure reaches quasi-instantaneously the so-called peak pressure $P_{\max }$ and then, it decays rapidly back to ambient pressure $P_{a}$ during the time $T$ (typically a few hundreds microseconds) of the so-called positive phase. This phase is often followed by the negative phase, during which the pressure is below the ambient pressure. Such pressure-time profile is usually modeled by the Friedlander function displayed in Figure 1 (see, e.g., [1-5]). Structure vulnerability studies and high explosive characterization need an accurate estimation of the pressure to compare with hydrocode numerical simulations (see, e.g., [6]).

In a typical air blast experimental setup, high-precision pressure sensors are used for measuring the pressuretime profile [7-8], and constant efforts are undertaken for enlarging the bandwidth of such sensors. Nowadays, commercially available piezoelectric sensors [9] can give signals with rise time of about 40 ns. Since sooner or later, the measurement of shorter rise times will be made possible, we address in this paper the issue of transmitting ultrafast signals delivered by wideband $(>10 \mathrm{MHz})$ pressure sensors.

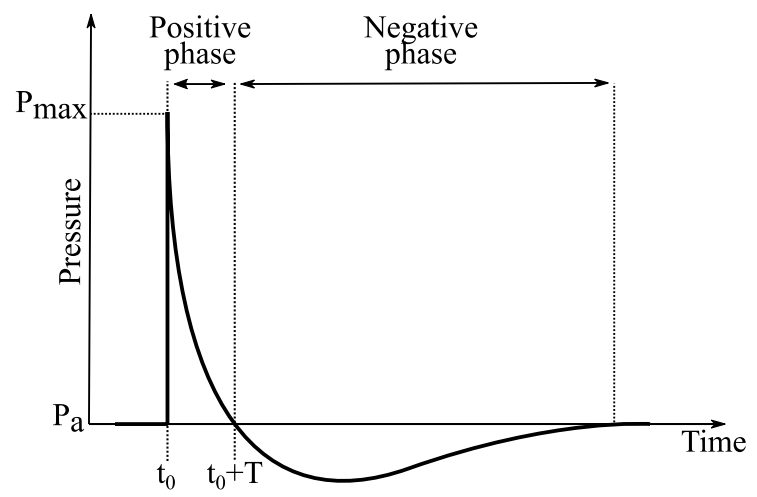

Figure 1. Profile of the Friedlander signal used for modeling the pressure generated by blast waves. $P_{\max }$ and $P_{a}$ denote respectively the peak pressure and the ambient pressure.

Long cables are commonly used during explosive detonation or air blast experiments for transmitting Friedlander-type signals from pressure sensors to the safe place of the acquisition unit (see, e.g., [10]). However, as shown in Section II, bandwidth limitation will make this standard and well-known wired technique unsuitable for transmitting over long distances. Moreover, extreme conditions involved during explosions (temperature over 1000 degrees Celsius with intense light, vibrations, ionized gases, etc.) may degrade the functionality of conventional wired measurement setups. 
In order to overcome the limitations of the wired technique for transmitting signals delivered by sensors during high explosive detonations, a wireless experimental setup is proposed in this paper. The voltage at the pressure sensor output is used here for the analog frequency-modulation of a radiofrequency carrier signal. The resulting frequency-modulated signal is next wirelessly transmitted to the acquisition unit, which proceeds to the digital demodulation. Very preliminary results investigating this solution were reported by us in [11]. It is shown here for the first time that the proposed wireless technique allows transmitting higher signal bandwidth than standard wired transmission setups and consequently, may be useful for remotely measuring Friedlander-type signals delivered by ultra-wideband sensors. Moreover, contrary to the wired setups, it is shown that the distance between sensors and the acquisition unit does not limit the bandwidth of the proposed wireless measurement setup.

The paper is organized as follows: Section II highlights the limitations of the wired setup commonly used for transmitting signals from the sensors output to the acquisition unit during air blast experiments. Section III details the design of the proposed wireless setup to overcome these limitations. From experimental results, Section IV validates that, contrary to the wired solution, the distance between sensors and the acquisition unit does not limit the measurement bandwidth of the wireless setup. Moreover, Section IV reports the dynamic measurement of blast pressure variations occurring within the fireball generated during a controlled explosion. Finally, Section $\vee$ gives the conclusion and proposes future work.

\section{Wired Setup for the Dynamic Measurement of Blast Pressure: Description and Limitations}

During an explosive detonation (see Figure 2), a shock wave is generated and moves supersonically, creating an overpressure.

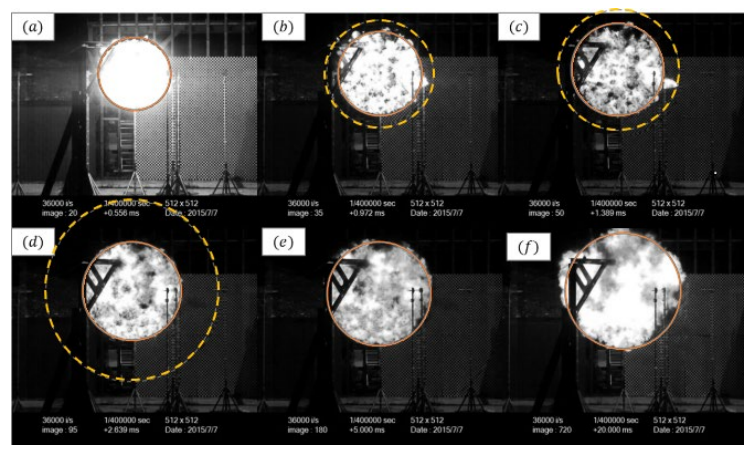

Figure 2. (a) The fireball during an explosion at various duration $t$ after explosive ignition: (a) $t=556 \mu s ;(b) t=972$ $\mu \mathrm{s}$; (c) $\mathrm{t}=1.389 \mathrm{~ms}$; (d) $\mathrm{t}=2.639 \mathrm{~ms}$; (e) $\mathrm{t}=5 \mathrm{~ms}$, and (f) $\mathrm{t}=20 \mathrm{~ms}$. The plain circle indicates the fireball expansion, while the dotted circle indicates the shock wave front.

Nowadays, for dynamically measuring the blast pressure during such an event, sensors (or gauges) are distributed around the charge to be characterized, and long coaxial cables $(>50 \mathrm{~m})$ are used for transmitting the Friedlandertype signal delivered by sensors to the safe place of an acquisition unit. The typical wired setup is shown in Figure 3. Piezo-electric pressure sensors with high output impedance $(\sim 1 \mathrm{G} \Omega)$ are often used for such experiment [10]. During the explosion, when the shock wave front impinges on the sensor, an amount of electric charge is produced at the sensor output, depending on the mechanical stress induced by the pressure on the piezoelectric crystal. The conditioning circuit converts the charge quantity into a voltage by using a common-drain Metal Oxide Semiconductor (MOS) amplifier. At the output port of this circuit, the impedance does not exceed $100 \Omega$ and a direct current (DC) voltage of typically few tens of Volts is generated due to the biasing current $I_{B I A S}$ of the amplifier. In order to avoid any current leakage, this $\mathrm{DC}$ voltage is filtered by using a series capacitor $C_{D C}$ of few $\mu \mathrm{F}$ (here, $C_{D C}=15 \mu \mathrm{F}$ ).

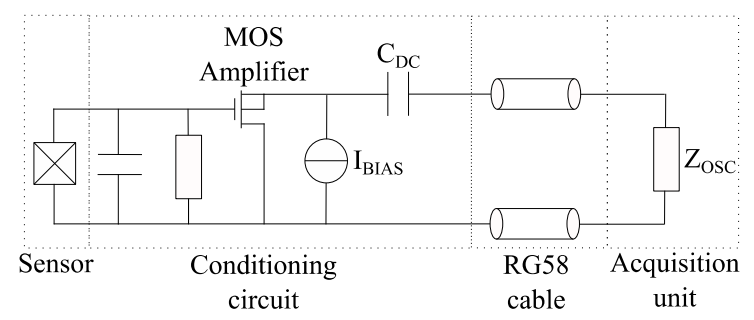

Figure 3. Standard wired setup for the dynamic measurement of the blast pressure. This setup allows the remote estimation of the aerial pressure peak $P_{\max }$ occurring just after the explosive ignition. $Z_{\text {OSC }}$ denotes here the input impedance of the digital oscilloscope used for the direct measurement of the baseband signal transmitted through long RG58 coaxial cable (typically of $50 \mathrm{~m}$ or more). 
The DC power unit of the sensor is usually located in a shelter close to the explosion (at a distance of about $10 \mathrm{~m}$ ). The RG58 coaxial cable of few tens of meters long (typically of $50 \mathrm{~m}$ or more) is commonly used for transmitting the baseband signal generated at the sensor output to the safe place of the acquisition unit. In this unit, the digital oscilloscope allows the direct measurement of the received signal. This conventional wired setup exhibits poorly documented issues for the dynamic measurement of the blast pressure and especially, for the high-precision measurement of the pressure peak occurring just after the explosion.

First, as the series capacitor $C_{D C}$ is connected to a long transmission line loaded by the high (but finite) input impedance of the digital oscilloscope $Z_{O S C}$ (typically $1 \mathrm{M} \Omega$ shunted by $10 \mathrm{pF}$ ), an undesirable high-pass filtering of the transmitted baseband signal occurs in the wired setup. If the corresponding cut-off frequency is not sufficiently low, the measured late temporal variation of the received signal may not reproduce the voltage at the sensor output. Furthermore, due to the shunt capacitance of transmission cables $(\sim 100 \mathrm{pF} / \mathrm{m}$ for the RG58 coaxial cable) combined with the input impedance of the digital oscilloscope, a low-pass filtering of the transmitted baseband signal occurs. Since the cut-off frequency of this low-pass filtering does not exceed in practice few tens of $\mathrm{MHz}$, the rejection of higher frequencies in the received signal takes place. The longer the coaxial cable, the lower the cut-off frequency and as a result, the narrower the transmission bandwidth of the wired setup. This undesirable effect of bandwidth reduction is illustrated in Figure 4. This latter displays the voltage at the output port of RG58 coaxial transmission cables, when a Heaviside step voltage of $1 \mathrm{~V}$ is applied at their input port. As expected, the longer the transmission cables, the higher the rise time of the output voltage (see Table 1). Such frequency bandwidth limitation is the major weakness of the conventional transmission line setup for transmitting over long distances the burst signals delivered by the wideband ( $>10 \mathrm{MHz}$ ) sensors.

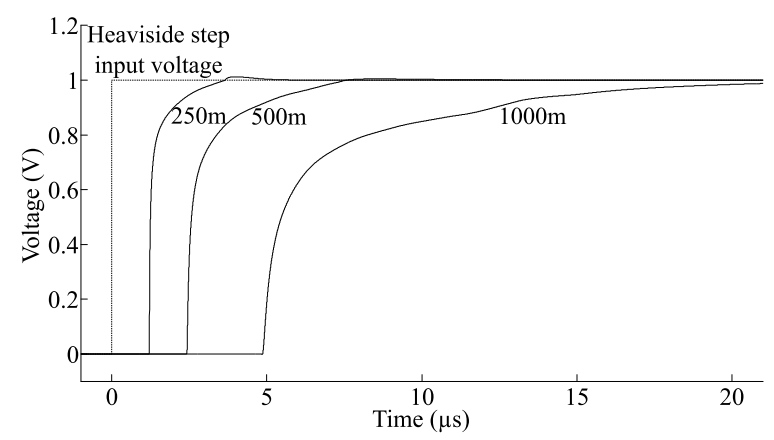

Figure 4: Simulated voltage at the output port of RG58 coaxial cables $\left(Z_{C}=50 \Omega\right.$ [12]) loaded by $Z_{\text {OSC }}(1 \mathrm{M} \Omega$ shunted by $10 \mathrm{pF}$ ) for different cable lengths between $250 \mathrm{~m}$ and $1000 \mathrm{~m}$, when the Heaviside step voltage of $1 \mathrm{~V}$ is applied at the input port. Simulations are performed by using Advanced Design System software.

Table 1. Rise time derived from Figure 4 of the output voltage as a function of the cable length. The rise time is defined as the time required by the voltage to reach for the first time $90 \%$ of the steady state value $(1 \mathrm{~V})$ from $10 \%$ of this value.

\begin{tabular}{ll}
\hline $\begin{array}{l}\text { Length of R58 } \\
\text { transmission cable }\end{array}$ & Rise time \\
\hline $250 \mathrm{~m}$ & $0.79 \mu \mathrm{s}$ \\
$500 \mathrm{~m}$ & $2.14 \mu \mathrm{s}$ \\
$1000 \mathrm{~m}$ & $7.40 \mu \mathrm{s}$ \\
\hline
\end{tabular}

Moreover, as the input impedance $Z_{O S C}(1 \mathrm{M} \Omega$ shunted by $10 \mathrm{pF})$ of the oscilloscope does not match the characteristic impedance $Z_{C}$ (about $50 \Omega$ over the bandwidth of interest) of the coaxial transmission lines, the transmitted baseband signal is reflected by the oscilloscope input impedance, and propagates back to the cable input. Next, this signal is reflected again, as the impedance $Z_{\text {in }}$ connected to the cable input differs generally from the characteristic impedance $Z_{C}$ of the coaxial line. Consequently, the steady state value is reached after signal roundtrips between input and output of the cable, whose duration depends on the cable length and level of impedance mismatches. For the sake of illustration, the voltage at the output port of RG58 coaxial cable is displayed in Figure 5 for $Z_{\text {in }}=47.5 \Omega$, when the Heaviside step voltage of $1 \mathrm{~V}$ is applied at the cable input. As shown in Table 2 , the longer the coaxial cable, the larger the time required by the output voltage to reach the steady state value. Such undesirable effect of inevitable impedance mismatches at the input and output ports of cables over the wide bandwidth of interest is the second major weakness of conventional wired setups for transmitting the aerial pressure signal delivered by ultra-wideband sensors. 


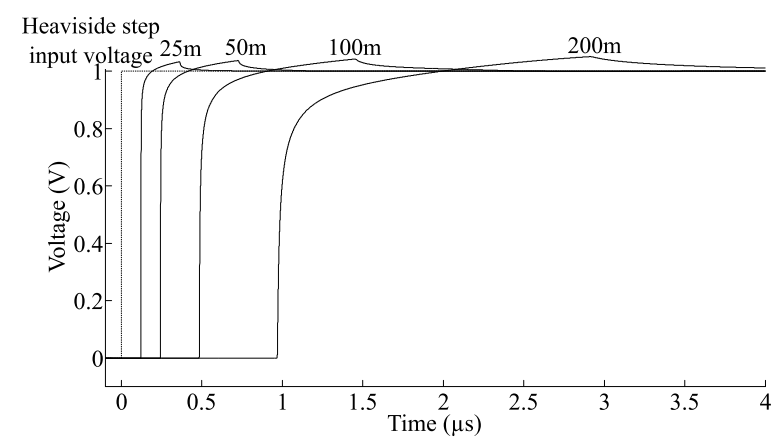

Figure 5: (a) Simulated voltage at the output port of RG58 coaxial cables coaxial cables $\left(Z_{C}=50 \Omega\right.$ [12]) loaded by $Z_{\text {OSC }}(1 \mathrm{M} \Omega$ shunted by $10 \mathrm{pF})$ for different cable lengths between $25 \mathrm{~m}$ and $200 \mathrm{~m}$, when the Heaviside step voltage of $1 \mathrm{~V}$ is applied at cable input port. Simulations are performed by using Advanced Design System software.

Table 2. Reaction time derived from Figure 5 as a function of the cable length. The reaction time is defined here as the time required by the output voltage to reach $\pm 2 \%$ of the steady state value.

\begin{tabular}{ll}
\hline $\begin{array}{l}\text { Length of R58 } \\
\text { transmission cable }\end{array}$ & Rise time \\
\hline $25 \mathrm{~m}$ & $251 \mathrm{~ns}$ \\
$50 \mathrm{~m}$ & $525 \mathrm{~ns}$ \\
$100 \mathrm{~m}$ & $1243 \mathrm{~ns}$ \\
$200 \mathrm{~m}$ & $2585 \mathrm{~ns}$ \\
\hline
\end{tabular}

Let the so-called Friedlander-type voltage $V_{F}(t)$ be applied at the input port of a transmission cable. This voltage is defined as follows:

$$
\begin{array}{rlrl}
V_{F}(t) & =V_{\max } \frac{t}{\tau} & \text { for } 0 \leq t \leq \tau \\
& =V_{\max }\left(1-\frac{t-\tau}{T}\right) e^{-\lambda \frac{t-\tau}{T}} \text { for } t>\tau
\end{array}
$$

Eq. (1) may be used to model the voltage delivered by a pressure sensor during an explosion, where $\tau$ denotes the time required by the voltage to reach its maximum value $V_{\max }, T(\gg \tau)$ is the duration of the positive phase, and $\lambda$ designates a positive constant. Figure 6 displays the simulated voltage at the output port of RG58 coaxial cables, when the voltage-time profile of Eq. (1) is applied at the input. As expected from the above analysis, the higher the cable length, the larger the discrepancies between the input and the output voltage profiles. Since the measurement of blast effects generated by explosives requires long distances (and therefore, long cables) for transmitting the sensor signals to the safe place of the acquisition unit, the derivation of the pressure peak $P_{\max }$ from the measurement of the peak voltage $V_{\max }$ may suffer from high inaccuracy.

In order to overcome the above-mentioned limitations of standard wired setups for the dynamic measurement of blast pressure signal, a wireless solution is proposed in the next Section.

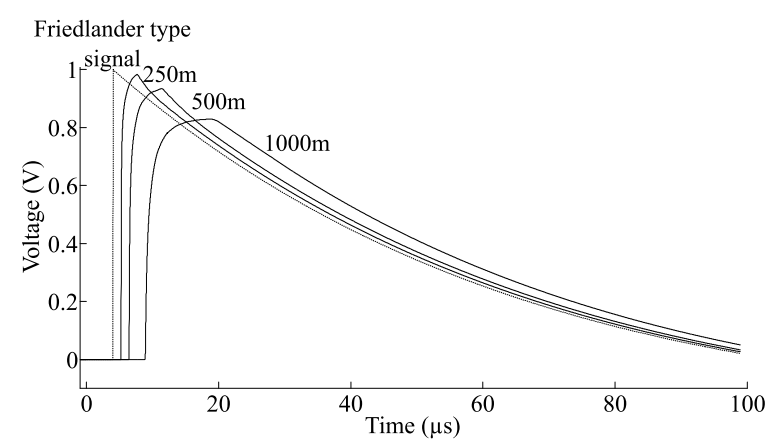

(a) 


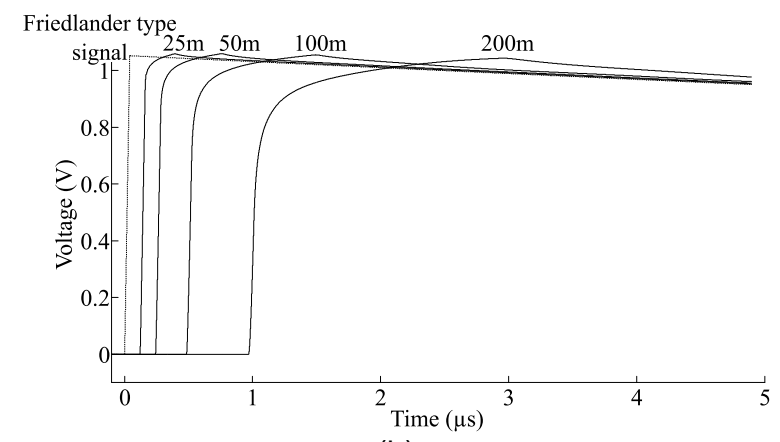

(b)

Figure 6: Simulated voltage at the output port of RG58 coaxial transmission cables, when the Friedlander-type voltage $V_{F}(t)$ defined by Eq. (1) is applied at the input port of the cables, with $V_{\max }=1 \mathrm{~V}, T=100 \mu \mathrm{s}, \lambda=1$ and $\tau=40 \mathrm{~ns}$ (using the commercial off-the-shelf pressure sensor reported in [9], $\tau$ may actually reach $40 \mathrm{~ns}$ ) and for different cable lengths : (a) between $250 \mathrm{~m}$ and $1 \mathrm{~km}$ and (b) between $25 \mathrm{~m}$ and $150 \mathrm{~m}$. The impedance $Z_{\text {in }}$ of the voltage source at the input of the cable is of $50 \Omega$, and simulations results are performed by using Advanced Design System software.

\section{Wireless Setup for the Dynamic Measurement of Blast Pressure Signal: Design Methodology}

\subsection{Operating principle}

To transmit the signal delivered by a pressure sensor during an explosive detonation or a blast experiment, the proposed wireless setup performs:

$i$. the analog frequency modulation of the radiofrequency (RF) carrier signal with the voltage $V_{\text {mod }}$ delivered at the output port of the sensor conditioning circuit (see Figure $7(\mathrm{a})$ ). Ideally, this voltage depends linearly on the pressure existing at the sensor location;

ii. the wireless propagation of the frequency-modulated (FM) signal through the propagation channel;

iii. the estimation of instantaneous frequencies embedded in the received FM signal from the digital microwave frequency counter (see Figure $7(\mathrm{~b})$ ). From these frequencies, the time-variation of the modulating voltage $V_{\text {mod }}(t)$ delivered at the output port of the conditioning circuit is estimated;

iv. Finally, knowing the sensitivity of the pressure sensor, the derived signal $V_{\text {mod }}(t)$ allows the estimation of the pressure profile $P(t)$ at the sensor location.

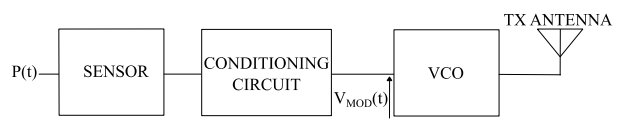

(a)

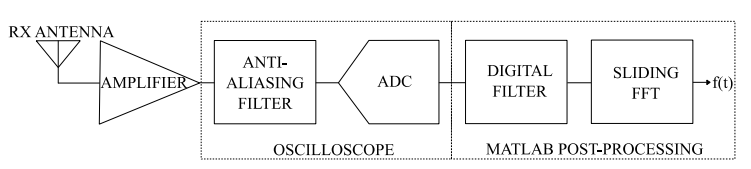

(b)

Figure 7: Block diagrams of the (a) transmission unit (Tx-unit) and the (b) reception unit (Rx-unit). The voltage $V_{\text {mod }}(t)$ indicated in the Tx-unit is called the modulating voltage, and is estimated at any time $t$ from the measurement in the Rx-unit of the instantaneous frequency $f(t)$ of the received frequency-modulated signal.

The Tx-unit converts the voltage variations $V_{\bmod }(t)$ at the output of the conditioning circuit into a frequency deviation of the RF carrier signal by using a Voltage Controlled Oscillator (VCO). The resulting analog FM signal is wirelessly transmitted to the Rx-unit by using the Tx-antenna. In the Rx-unit, the received FM signal is first amplified and then low-pass filtered in order to remove eventual aliasing spectrum effect. The sampling is performed by using an Analog-to-Digital Converter (ADC) device, and the variation of the instantaneous radio-frequency $f(t)$ of this signal at any time $t$ is derived from Fast Fourier Transform (FFT).

\subsection{Calibration process}

The VCO device in the Tx-unit converts ideally a voltage variation $\Delta V_{\text {mod }}$ into the instantaneous frequency shift $\Delta f=K_{0} \Delta V_{\text {mod }}$, where $K_{0}$ is called the sensitivity coefficient of the VCO. In practice, this coefficient is not constant 
and depends, for example, on the ambient temperature [13]. Hence, the calibration of the wireless setup is required for taking into account the eventual deviation from the ideal linear conversion law.

The proposed calibration process is driven as follows: before the explosive detonation, a controlled periodic voltage $V_{\text {cal }}(t)$ is applied at the input port of the VCO device (a saw tooth signal is used in this work). The resulting FM signal is wirelessly transmitted through the propagation channel to the Rx-unit, and the instantaneous frequency $f_{\text {cal }}(t)$ of the received FM signal is measured. Since the calibration voltage $V_{c a l}(t)$ is periodic, each given voltage $V_{c a l}$ is applied many times to the VCO input during the calibration process. Due to electronic noise, identical (or very close) voltages do not generate identical $f_{c a l}$. The polynomial regression of $f_{c a l}$ on $V_{c a l}$ is performed in this study. At the end of the calibration process, any modulating voltage $V$ applied at the input of the VCO can be remotely estimated from the measurement of the instantaneous frequency $f$ embedded in the received FM signal.

\subsection{Key descriptors of the wireless transmission setup and design methodology}

One key descriptor of the proposed wireless solution is the $3 d B$ bandwidth of the setup. This bandwidth is mainly limited by three following components: (1) the sensor bandwidth $B_{S}$, (2) the bandwidth of the VCO device $B_{V C O}$ and, (3) the bandwidth of the digital microwave frequency counter $B_{c o u n t}$. The bandwidth $B_{V c o}$ is required to include $B_{S}$, and the output impedance of the conditioning circuit must match the input impedance of the VCO device. Moreover, the bandwidth $B_{F M}$ of the transmitted FM signal, which is given by Carson rule $2 B_{S}\left(1+m_{0}\right)$ where $m_{0}$ denotes the modulation rate [14, 15], must be included in the bandwidth of all RF devices of the wireless setup. Furthermore, the amplitude of the received FM signal must be amplified for taking advantage of the maximal full-scale range offered by the ADC device. After amplification, the received $\mathrm{FM}$ signal is sampled with a sampling rate $f_{\text {samp }}$ which must be higher than $2 f_{0}$ to satisfy the Shannon criterion, where $f_{0}$ denotes the RF carrier frequency.

In order to measure the instantaneous frequency $f\left(t_{i}\right)$ in the received FM signal at any time $t_{i}$, a time-windowing of duration $T_{\text {meas }}\left(<1 / 2 B_{S}\right)$ and starting at time $t_{i}$, is performed to the signal. Next, FFT is applied to the windowed signal for deriving the set of spectral amplitudes for $N=T_{\text {meas }} f_{\text {samp }}$ frequencies. Following [16], we assume here that the frequency for which the spectral amplitude of FFT takes its highest value provides the estimation of the instantaneous frequency $f\left(t_{i}\right)$ in the FM signal at time $t_{i}$. Since the frequency resolution of the FFT does not exceed $1 / T_{\text {meas }}=f_{\text {samp }} / N$, and as the principal source of noise is the quantization error variance $\sigma_{q}^{2}$ of the ADC device $\left(\sigma_{q}^{2}=q^{2} / 12\right.$, where $q$ is the quantization error given by $\left.2 V_{M} / 2^{N_{D}}-1\right)$, it can be derived from [16] that the standard deviation of the estimated instantaneous frequency is lower bounded by $\sigma_{\min }$ given as follows:

$$
\sigma_{\min }=\frac{2^{-N_{D}}}{\pi} \frac{V_{M}}{V_{R F}} \frac{f_{\text {samp }}}{\sqrt{N\left(N^{2}-1\right)}}
$$

where $N_{D}$ and $V_{M}$ denote, respectively, the effective digital resolution and the maximum input voltage of the ADC device, and $V_{R F}$ designates the voltage applied to the input of ADC device. In practice, $N$ should be then chosen so that $\sigma_{\min }$ is close to the standard deviation of the calibration signal $V_{\text {cal }}(t)$. Moreover, by applying the zero-padding technique [14], which consists of adding a number $Z$ of zero-valued samples in the time-windowed FM signal, the number of frequency samples is finally given by $N+Z$. This technique does not enhance the frequency resolution of the ADC, but it allows improving the resolution of the FFT. The number $Z$ should be then chosen so that $f_{\text {samp }} /(N+Z) \approx \sigma_{\min }$. Finally, it can be observed from Eq. (2) that the measurement resolution of the instantaneous frequency at any time $t_{i}$ may be enhanced by:

i. improving the effective digital resolution of the ADC device;

ii. enlarging the bandwidth of the digital microwave frequency counter $B_{\text {count }}$ from which the measurement duration -or equivalently, the number of frequency samples in the FFT- can be derived;

iii. increasing the received RF power $P_{R X}$ for rising the voltage applied to the input of ADC device.

Moreover, the lowest RF power $P_{R X_{M I N}}$ required at the ADC input, called the sensitivity of the ADC, depends on the minimum carrier-to-noise power ratio $C N R_{M I N}$ required for ensuring the FM demodulation. $C N R_{M I N}$ is found to be about 10, whatever the modulation rate [17]:

$$
C N R_{M I N}=\frac{P_{R X}}{P_{N O I S E}} \cong 10
$$

where $P_{\text {NOISE }}$ denotes the noise power in the carrier signal. This power is estimated from the quantization error variance $\sigma_{q}^{2}$ of the ADC device. From Eq.(3), the sensitivity of ADC device is then derived as follows:

$$
P_{R X_{M I N}}=\frac{10}{3} 2^{-2 N_{D}} V_{M}^{2}
$$

Consequently, according to the link budget based on Friis Equation (see, e.g., [18]), the received power $P_{R X}$ is higher than the ADC sensitivity when the transmitted power $P_{T X}$ is larger than $P_{T X_{M I N}}$ given by:

$$
P_{T X_{M I N}}=\frac{P_{R X_{M I N}}}{\rho G_{\text {ampli }} G_{T X} G_{R X} G_{\text {propa }}}
$$


where $G_{T X}$ and $G_{R X}$ are the gains of respectively the Tx-and Rx-antennas, $G_{p r o p a}$ is the path loss in the propagation channel at the carrier frequency $f_{0}, G_{\text {ampli }}$ is the gain of the RF power amplification in the Rx-unit (see the block diagram of Fig. 7(b)), and $\rho$ is the efficiency due to the eventual polarization mismatch between the Tx- and Rxantennas.

\section{Wireless and Analog RF Setup for the Dynamic Measurement of Blast Pressure Signal: Experimental Validation}

Figure 8 shows the block diagrams of the experimental setup which was designed for transmitting Friedlander-type signals over long distances, while Table 3 reports the key descriptors of this setup derived from the design methodology detailed in Section III.

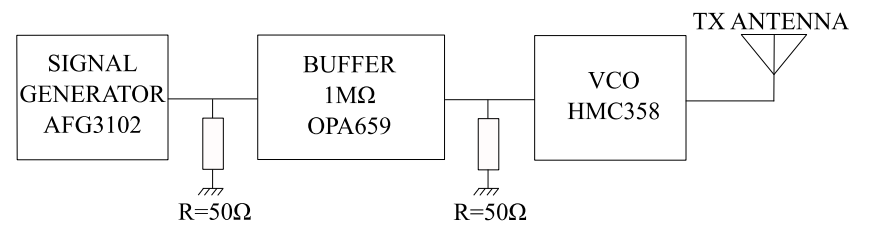

(a)

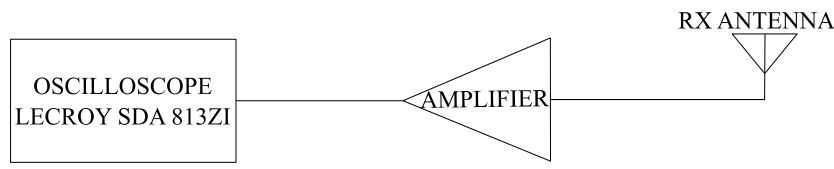

(b)

Figure 8: Block diagrams of the experimental setup used for wirelessly transmitting Friedlander-type signals over long distances: (a) Tx-unit, and (b) Rx-unit.

Before transmitting signals of interest, the calibration voltage $V_{c a l}(t)$ shown in Figure $9(a)$ is used as the modulating voltage in the Tx-unit. For each voltage $V_{c a l}$ at time $t$, the instantaneous frequency $f_{c a l}$ is estimated at a distance of $140 \mathrm{~m}$. As displayed in Figure 9(b), the resulting scattered plot of $f_{c a l}$ versus $V_{c a l}$ is obtained and the linear regression of $f_{c a l}$ on $V_{c a l}$ is performed (we assume here that uncertainty on voltage is negligible compared with one on the frequency). The regression law $f_{c a l}=\alpha+\beta V_{\text {cal }}$ is then derived, with $\alpha=5.55 \mathrm{GHz}$ and $\beta=0.12 \mathrm{GHz} / \mathrm{V}$ with the coefficient of determination $R^{2}$ of 0.9997 . Therefore, any voltage $V_{\text {mod }}$ applied at the input of the VCO device can be remotely estimated from the measurement of instantaneous frequency $f$ and the application of the relationship: $V_{\text {mod }}=(f-\alpha) / \beta$.

Table 3: Key descriptors of the wireless setup of Figure 8.

Descriptor of transmitted FM signals

\begin{tabular}{|c|c|}
\hline carrier frequency $\left(f_{0}\right)$ & $5.6 \mathrm{GHz}$ \\
\hline modulation rate $\left(m_{0}\right)$ & about $2 \%$ \\
\hline bandwidth of the signal generator $\left(B_{S}\right)$ & $100 \mathrm{MHz}$ \\
\hline bandwidth of the transmitted FM signal $\left(B_{F M}\right)$ & $200 \mathrm{MHz}$ \\
\hline \multicolumn{2}{|l|}{ Descriptor of the FM demodulation } \\
\hline duration of time-windowing $\left(T_{\text {meas }}\right)$ & $4 \mathrm{~ns}$ \\
\hline sampling rate of the ADC device $\left(f_{\text {samp }}\right)$ & $40 \mathrm{GS} / \mathrm{s}$ \\
\hline $\begin{array}{l}\text { number of samples in the time-windowed FM } \\
\text { signal }(N)\end{array}$ & 160 \\
\hline $\begin{array}{l}\text { number of zero-valued samples in the time- } \\
\text { windowed FM signal }(Z)\end{array}$ & $2^{18}$ \\
\hline effective digital resolution of ADC device $\left(N_{D}\right)$ & 5 \\
\hline full-scale voltage of the ADC device $\left(V_{M}\right)$ & about $1 \mathrm{~V}$ \\
\hline $\begin{array}{l}\text { bandwidth of digital microwave frequency } \\
\text { counter }\left(B_{\text {count }}\right)\end{array}$ & $100 \mathrm{MHz}$ \\
\hline sensitivity of ADC device $\left(P_{R X M I N}\right)$ & $5 \mathrm{dBm}$ \\
\hline \multicolumn{2}{|l|}{ Powers and gains } \\
\hline gain the Tx-antenna $\left(G_{T X}\right)$ & $16 \mathrm{dBi}$ \\
\hline gain the Rx-antenna $\left(G_{R X}\right)$ & $16 \mathrm{dBi}$ \\
\hline
\end{tabular}




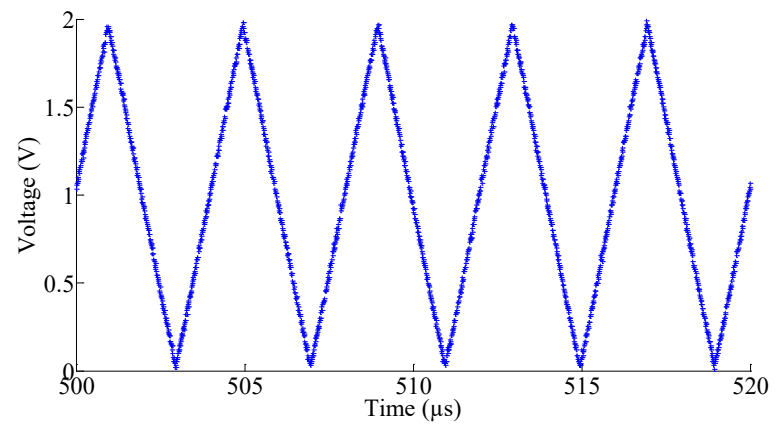

(a)

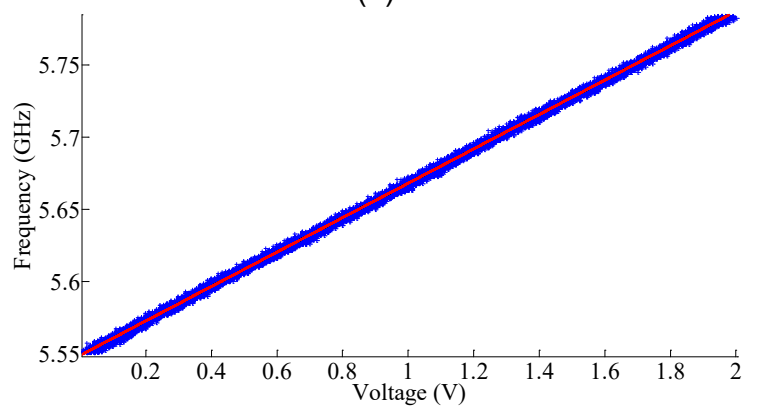

(b)

Figure 9: (a) Portion (from $\mathbf{5 0 0} \boldsymbol{\mu s}$ to $\mathbf{5 2 0} \boldsymbol{\mu s}$ ) of the signal used for calibration voltage $\boldsymbol{V}_{\text {cal }}(\boldsymbol{t}$ ), with period of $\mathbf{4} \boldsymbol{\mu s}$ and total duration of $\mathbf{2} \mathbf{~ m s}$; (b) transmitted instantaneous frequency as a function of the calibration voltage $\boldsymbol{V}_{\boldsymbol{c a l}}$ (in blue). The frequencies embedded in the transmitted signal are measured at the distance of $\mathbf{1 4 0} \mathbf{m}$ from the Txantenna. The linear regression (in red) is used here for deriving the regression of $\boldsymbol{f}_{\text {cal }}$ on $\boldsymbol{V}_{\boldsymbol{c a l}}$.

Once the calibration process is complete, the Heaviside step voltage of amplitude $1 \mathrm{~V}$ and rise time $5 \mathrm{~ns}$ is used as the modulating voltage $V_{\text {mod }}(t)$. For comparison purpose, the same step voltage is simultaneously applied at the input of the $100 \mathrm{~m}$ long RG58 coaxial cable. Figure 10 displays the voltage received by using the wired and wireless setups. It can be observed that:

$i$. When using the wired setup, the measured rise time $(10 \%$ to $90 \%)$ of the received voltage is $(100 \pm 6) n s$ (with a $95 \%$ confidence interval) and consequently, is found significantly larger than the rise time ( $5 \mathrm{~ns}$ ) of the step voltage applied at the cable input. As highlighted in Section II, the reason of this discrepancy originates in the lowpass filtering of the transmitted baseband signal due to the shunt capacitance $(\sim 100 \mathrm{pF} / \mathrm{m})$ of the RG58 coaxial cable and to the input impedance $(1 \mathrm{M} \Omega$ shunted by $16 \mathrm{pF})$ of the digital oscilloscope. Moreover, the time required by the output voltage to reach $\pm 2 \%$ of the steady state value is $1.15 \mu$ s. As discussed in Section II, this long reaction time originates in the impedance mismatches at the input and output ports, despite the use of a signal generator of output impedance $Z_{\text {OUT }}=50 \Omega$;

ii. When using the wireless setup, the measured rise time (about $10 \mathrm{~ns}$ ) of the signal received at a distance of $140 \mathrm{~m}$ is found to be significantly shorter than the one obtained from the wired setup. Moreover, as expected, the time required by the output voltage to reach $\pm 2 \%$ of the steady state value is very short $(<28 \mathrm{~ns})$.

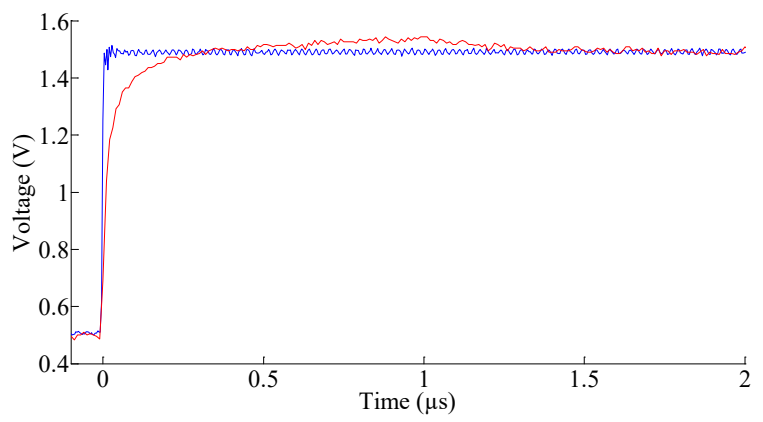


Figure 10: Heaviside step voltage of $1 \mathrm{~V}$ (from $0.5 \mathrm{~V}$ to $1.5 \mathrm{~V}$ ) and rise time of $5 \mathrm{~ns}$ received by using the wired setup (in red) and the proposed wireless setup (in blue). The wireless transmission is carried out over $\mathbf{1 4 0} \mathbf{~ m}$, while the wired transmission uses a RG58 cable of $100 \mathrm{~m}$ long loaded by the input impedance $Z_{\text {osC }}$ ( 1 M $\Omega$ shunted by $10 \mathbf{p F}$ ) of the serial data analyzer LECROY SDA $813 Z$.

Another experimental demonstration of the benefit of the proposed wireless solution compared with the wired setup consists of transmitting the Friedlander-type signal with $T=100 \mu$ s and $\tau=5 \mathrm{~ns}$. This signal, generated by using the programmable Tektronix AFG3102, is used here to simulate the voltage delivered by an ideal ultrawideband pressure sensor located at $50 \mathrm{~cm}$ of a $500 \mathrm{~g}$ Semtex explosive. It can be observed from Figure 11 that the signal received by the wired setup requires $1 \mu$ s to reach its peak value, while only $10 \mathrm{~ns}$ are needed when using the wireless setup. Consequently, the wireless setup allows a time of arrival and reaction time of the shock wave estimation with higher measurement accuracy than the one provided by the wired setup. Moreover, contrary to the wired setup, the proposed wireless solution gives the direct measurement of the peak value $V_{\max }$, without requiring any post-processing on measurement data (see, e.g., [4] for details on the limitations of such post-processing usually applied on the measurement data for estimating the peak value $V_{\max }$ in wired setups). Furthermore, the effect of the impedance mismatches at the input and output ports of the coaxial transmission cable is clearly apparent in Figure 11, and negatively impacts the measurement accuracy of $V_{\max }$. This issue related to impedance mismatches does not arise in the wireless setup.

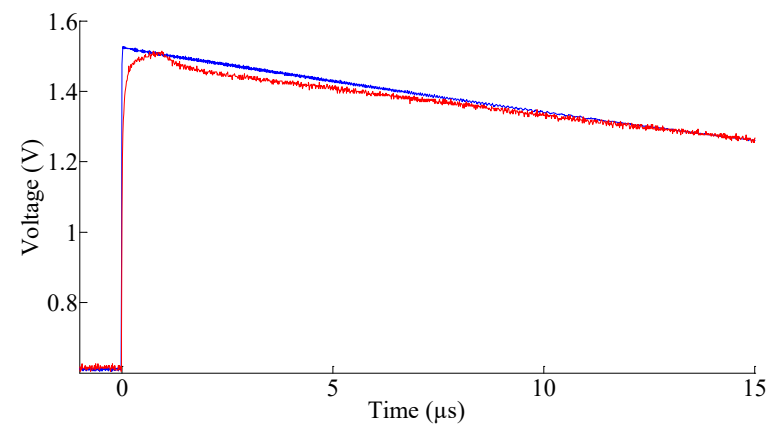

Figure 11: Friedlander-type signal with $\boldsymbol{T}=\mathbf{1 0 0} \mu \mathrm{s}$ and $\boldsymbol{\tau}=\mathbf{5} \mathrm{ns}$, received by the wired setup at a distance of $100 \mathrm{~m}$ (in red), and received at a distance of $140 \mathrm{~m}$ by the proposed wireless setup (in blue). During the calibration process, a polynomial of order $\mathbf{3}$ was used for the regression of $\boldsymbol{f}_{\boldsymbol{c a l}}$ on $\boldsymbol{V}_{\boldsymbol{c a l}}$.

In the last but not least experiment, the wireless setup is used to transmit and receive the pressure signal delivered by the piezoelectric PCB $113 \mathrm{~A} 34$ sensor [19] during an explosion. The sensor is located at $50 \mathrm{~cm}$ of aluminized TriNiTrotoluene (TNT) explosive of $600 \mathrm{~g}$ (see Figure 12). When the sensor, VCO device (located at $1 \mathrm{~m}$ from the explosive) and Tx-antenna are inside the fireball, it is demonstrated here that the carrier frequency of $(5.3 \pm 0.1) \mathrm{GHz}$ chosen in this last experiment allows the wireless transmission of the pressure signal with very low distortion. In Table 4 are summarized the key descriptors for this experiment. The other descriptors are reported in Table 3 . The distance between the Rx- and Tx-antennas is set to about $30 \mathrm{~m}$. The explosion occurs $65 \mu \mathrm{s}$ after the end of the calibration signal.

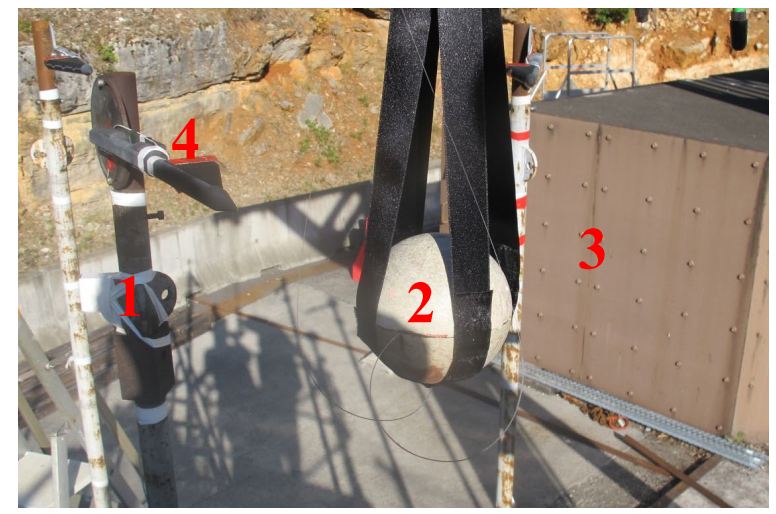

Figure 12: Photograph of the experimental setup (detail). The piezoelectric PCB $113 A 34$ sensor (4) is placed at $50 \mathbf{~ c m}$ of aluminized TriNiTrotoluene (TNT) spherical explosive charge of $600 \mathbf{g}$ (2). The VCO device (HMC587) and Tx-antenna (half-wave dipole) (1) are located at $\mathbf{1} \mathbf{~ m}$ from the center of the spherical charge. The rest of the Tx-unit is placed inside the bunker (3). The acquisition unit and Rx-unit are placed in a second bunker (not shown here) located at $\mathbf{3 0 ~} \mathbf{m}$ from the charge. 
For comparison purpose, the pressure signal delivered by the sensor is also applied at the input of a RG58 coaxial cable of $60 \mathrm{~m}$ long. Figure 13 displays the pressure obtained from the signal received by the wired and wireless setups (the pressure is derived here from multiplying output sensor voltage by the sensor sensibility of about $73 \mathrm{mV} /$ bar [19]). The VCO and its antenna are inside the fireball generated by the explosion from at most $300 \mu \mathrm{s}$ after the peak overpressure. The wired and wireless estimations of pressure are found in very good agreement, and both the wired and wireless setups require $4 \mu \mathrm{s}$ for reaching the pressure peak of $35.3 \mathrm{bar}$. In this experiment the bandwidth is not limited by the transmission channel, but mainly by the sensor bandwidth. As the shock wave front is here normally incident upon the PCB $113 \mathrm{~A} 34$ sensor surface, this bandwidth is found to be of about $100 \mathrm{kHz}$.

Table 4: Key descriptors of the wireless setup for the experiment with aluminized TNT explosive of $600 \mathrm{~g}$.

\begin{tabular}{|l|c|}
\hline $\begin{array}{l}\text { bandwidth of the transmitted FM signal } \\
\left(B_{F M}\right)\end{array}$ & $100 \mathrm{kHz}$ \\
\hline duration of time-windowing $\left(T_{\text {meas }}\right)$ & $50 \mathrm{~ns}$ \\
\hline sampling rate of the ADC device $\left(f_{\text {samp }}\right)$ & $20 \mathrm{GS} / \mathrm{s}$ \\
\hline gain of TX-antenna $\left(G_{T X}\right)$ & $2 \mathrm{dBi}$ \\
\hline gain of Rx-antenna $\left(G_{R X}\right)$ & $16 \mathrm{dBi}$ \\
\hline Transmiited power & $4 \mathrm{dBm}$ \\
\hline
\end{tabular}

This experiment demonstrates that the wireless transmission of the pressure is possible through the fireball of the explosion, even if some unexpected spikes are detected (see, e.g., at time $t=45 \mu$ s in Figure 13). These spikes (whose interpretation remains to be found) could be eventually removed by enhancing the radiofrequency link budget. This could be easily done by adding a power amplifier in the Tx-unit.

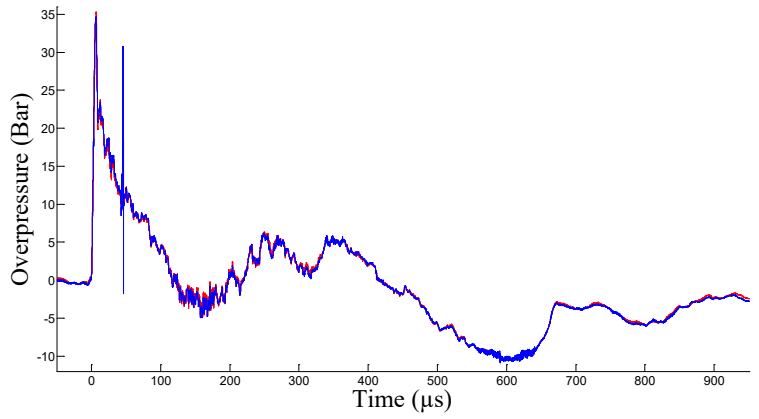

Figure 13: Overpressure as a function of time obtained from the signal received (1) by the wired setup (in red) and (2) by the wireless setup (in blue). During $300 \mu \mathrm{s}$, the VCO and the Tx-antenna are inside the fireball generated by the explosion when wirelessly transmitting this pressure signal. A polynomial of order $\mathbf{3}$ was used in the calibration process for the regression of $\boldsymbol{f}_{c a l}$ on $\boldsymbol{V}_{\boldsymbol{c a l}}$. This order is required due to the non-linearity of the VCO response in the large full-scale range of the measured pressure.

Compared with the state-of-the-art [9], the piezoelectric PCB 113A34 sensor used in this last experiment has a low reaction time of few $\mu$ s and consequently, cannot highlight the benefit of the wireless setup compared with the wired setup for short transmission channel of few ten meters. However, this sensor demonstrates that the calibration performed few microseconds before the fireball formation, with a propagation channel different, is still usable when the VCO is inside the fireball.

\section{Conclusion}

A wireless setup based on the analog frequency-modulation technique and using a digital frequency microwave meter was proposed for transmitting and receiving signal variations delivered by pressure sensors. It allows measuring the blast pressure within a fireball generated by an explosion.

Two experiments have demonstrated that, contrary to the wired solution, the distance between the sensor and the acquisition unit does not limit the measurement bandwidth of the wireless setup. This makes the reported wireless technique suitable for transmitting ultrafast variations of pressure inside the fireball of explosions.

The two main drawbacks associated with the proposed wireless set up are the following ones:

(1) The extension of the approach for the remote analysis of explosive detonation occurring in cluttered environments is not straightforward. As a matter of fact, electromagnetic propagation with multi-path and multiple reflections may degrade the signal-to-noise ratio and consequently, cause a disadvantageously impact on the measurement accuracy of the overpressure peak;

(2) Due to the limited electromagnetic susceptibility \& immunity of electronic devices in the wireless setup, the electromagnetic power level radiated by the sensors must be sufficiently low in order to avoid any eventual malfunction in these devices. This constraint limits the separation distance between sensors and the reader to few tens meters in practice. This highest achievable distance may be not sufficient for some long-range applications. 
Ongoing works are devoted to the development of a wireless solution based on the direct sampling of the received FM signal and the application of the so-called direct detection technique. Moreover, frequency bandwidth about $1 \mathrm{GHz}$ is, at least in principle, achievable by using the proposed wireless architecture. Thanks to large bandwidth, multiple FM signals may be simultaneously transmitted and processed using the proposed wireless technique. Extension of this work to the simultaneous wireless transmission of FM signals from multiple sensors is in progress.

\section{Acknowledgements}

The authors thank Fabrice Mathieu for the help during the development of the high bandwidth conditioning circuit and the Occitanie Regional Council in France for contributing to the financial support of this work.

\section{References}

[1] W.E. Baker, Explosions in Air, University of Texas Press, 1973.

[2] M. Ismail, S. G. Murray, Study of the Blast Wave Parameters from Small Scale Explosions, Propellants, Explosives, Pyrotechnics 1993,18, 11-17.

[3] S.A. Formby, R.K. Wharton, Blast characteristics and TNT equivalence values some commercial explosives detonated at ground level, J. of Hazardous Materials 1996, 50, I83- 198.

[4] M. D. Goel, V. A. Matsagar, A. K. Gupta, S. Marburg, An Abridged Review of Blast Wave Parameters, Defence Science Journal 2012, 62, 300-306.

[5] S . E. Rigby, A. Tyas, S. D. Fay, S. D. Clarke, J. A. Warren, Validation of semi-empirical blast pressure predictions for far field explosions - is there inherent variability in blast wave parameters?, 6 th International Conference on Protection of Structures against Hazards, October 16-17 2014, Tianjin, China.

[6] W. Fickett, C. William, Detonation: Theory and Experiment, Dover Publications, 2011.

[7] N. Chaudhary, Pressure Sensors for Blasts and Shock Waves: State of Art, Int. J. of Advanced Research in Physical Science, 2015, Vol. 2

[8] F. Bauer, PVDF shock sensors: applications to polar materials and high explosives, IEEE Trans. on Ultrasonics, Ferroelectrics, and Frequency Control, 2000, 47,1448 - 1454

[9] Piezoelectric transducers for high frequency measurements, accessed on Dec. 10, 2019. [Online]. Available: https://mueller-instruments.de/fileadmin/Downloads/englische medien/Mueller Pressure Sensor M.pdf

[10] L.P. Silver, Evaluation of Air Blast Measurement Techniques, 75th Shock and Vibration Proceedings, Virginia Beach, VA, October 17 - 22, 2004.

[11] J. Fourmann, A. Coustou, H. Aubert, P. Pons, J. Luc, A. Lefrançois, M.Lavayssière, A.Osmont, Wireless Sensors for the Incident Pressure Measurement in Air Blast, European Microwave Week, London, UK, Oct 2016.

[12] PRO-POWER, RG58 Coaxial Cable, RG58 Coaxial Cable datasheet, Aug. 2016 V1.2.

[13] Analog Device, MMIC VCO w/ BUFFER AMPLIFIER, 5.8 - 6.8 GHz14] S. Smith, "The Scientist \& Engineer's Guide to Digital Signal Processing", California Technical Pub., 1997.

[14] S. Smith, The Scientist \& Engineer's Guide to Digital Signal Processing, California Technical Pub., 1997.

[15] J. R. Carson, Notes on the theory of modulation, Proceedings of the IEEE, 1963, 51, 893-896.

[16] A. C. Rife, R. R. Boorstyn, Single-Tone Parameter Estimation from Discrete-Time Observations, IEEE Trans. Inf. Theory, 1974, 20.

[17] A. Wojnar, Exact Threshold Location in Analog FM Systems, Proc. IEEE, 1970, 58, 251-253.

[18] C.A. Balanis, Antenna Theory: Analysis and Design 3rd ed., Hoboken, NJ: John Wiley, 2005.

[19] Model 113A34 High frequency Invar ICP® pressure probe, 1000 psi, 5 mV/psi, 0.218"' dia. Installation and Operating Manual. [Online] www.pcb.com 